\title{
From Singularity Theory to Finiteness of Walrasian Equilibria
}

\author{
Sofia B. S. D. Castro ${ }^{\diamond}$ Sami Dakhlia* P. B. Gothen \\ sdcastro@fep.up.pt sami.dakhlia@esce.fr pbgothen@fc.up.pt
}

$\diamond$ CMUP and Faculdade de Economia do Porto, Universidade do Porto, Rua Dr. Roberto Frias, 4200-464 Porto, Portugal.

^ Ecole Supérieure du Commerce Extérieur, PULV, 92916 Paris - La Défense, France. Corresponding author.

- CMUP and Faculdade de Ciências, Universidade do Porto, Rua do Campo Alegre, 4169-007 Porto, Portugal.

\begin{abstract}
The paper establishes that for an open and dense subset of smooth exchange economies, the number of Walrasian equilibria is finite. In particular, our results extend to non-regular economies; it even holds when restricted to the subset of critical ones. The proof rests on concepts from singularity theory.
\end{abstract}

Keywords: Arrow-Debreu, transversality, exchange economy, critical equilibria, finite singularity type

JEL classification: D50, C62, C02

First and third authors partially supported by FCT (Portugal) through the Centro de Matemática da Universidade do Porto. 


\section{Introduction}

We establish that generically, the set of equilibria of any economy, whether regular or not, is finite. By generically, we mean that this property holds for an open and dense subset of economies in the $C^{\infty}$ Whitney topology (and a residual, therefore dense, subset in the $C^{\infty}$ compact-open topology). Moreover, our results hold restricted to critical economies, showing that even the generic critical economy has a finite number of equilibria. This extension to non-regular economies adds to a long series of contributions on local isolation, determinacy, and other generic properties of the set of equilibrium prices, spawned by Debreu's seminal 1970 paper. It also extends results by Allen (1984) and Mas-Colell and Nachbar (1991) and proves conjectures found in the latter.

Debreu (1970) establishes that for all but a Lebesgue measure zero subset of endowments, Arrow-Debreu economies are regular and thus have locally isolated, indeed finitely many equilibria. Despite its fundamental nature, the full extension of this result to critical economies has so far been elusive. The proof of finiteness in a strong generic sense for both regular and critical economies would not only confirm heretofore unproven conjectures, but also further characterize the equilibrium manifold.

If the equilibrium correspondence is countably-valued, a continuous random selection exists, which, in turn, has application to the question of existence of equilibrium in Cournot economies (see Allen, 1994). Finiteness (and the existence of a continuous random selection) is required to perform sensitivity analysis for computable general equilibrium models when multiple equilibria are present (see Berliant and Dakhlia, 2002).

Allen (1984) and Mas-Colell and Nachbar (1991) make contributions in this direction. Allen (1984) works with economies parametrized on a compact manifold with boundary and establishes finiteness of equilibria for a residual subset of parameters in both the $C^{\infty}$ Whitney and compact-open topologies. Her proof resorts to results in differential topology applied directly to smooth $\left(C^{\infty}\right)$ aggregate excess demand (henceforth, AED) functions rather that the deeper primitives of preferences and endowments.

Mas-Colell and Nachbar (1991), on the other hand, work directly with preferences and endowments and use no more than transversality to obtain countability (though not finiteness) of equilibria for a residual subset of utility functions of the first consumer in the $C^{\infty}$ compact-open topology. They conjecture (pp. 402-403) that the results can be strengthened in two ways, 
namely that finiteness (rather than mere countability) should hold for opendense (rather than merely residual) sets, but do point to the mathematical subtleties associated with the study of the set of zeros in the neighborhood of a critical zero.

A possible strategy for a transversality-based argument requires that the economy be perturbed so as to transform its AED into a function with isolated zeros. The challenge of this type of exercise is then to relate the perturbed AED to the deeper primitives of preferences and endowments. Results recently established by Castro et al. (2010), allow us to do just this and perturb AED directly. ${ }^{1}$ It is in this spirit that Castro and Dakhlia (2008) obtain finiteness by establishing that, generically, AED is Thom-Boardman stratified. While their approach has the advantage of being geometrically intuitive, their result ultimately only holds for analytic functions.

In this paper, we do not focus on regularity, since by definition it excludes critical economies, but rather on whether AED functions are of Finite Singularity Type (FST). ${ }^{2}$ The FST concept is first defined by du Plessis in Gibson et al. (1976, Definition III, 2.7) and has been used strictly in the context of singularity theory, which may be why it has so far not drawn economists' attention. Although its formulation is straightforward, the concept is quite subtle. We apply it to the case of non-parametrized economies to convey the essential ideas in the clearest possible way. The application of our approach to explicitly parametrized economies is a natural, though non-trivial, problem to which we hope to come back in the future.

In short, our approach is thus as follows: (1) perturb AED directly (knowing that such a perturbation is equivalent to a perturbation of a single consumer's preferences); (2) establish that, generically (even when restricting to the space of critical maps), AED functions are of FST.

After a section establishing notation and some preliminary results, we proceed with two technical sections. While they may be skimmed by readers familiar with either differential topology (section 3) or singularity theory (section 4), they are included here for completeness and ease of reference. They also contain new results of a strictly technical nature. Section 5 contains the results that lead to the proof of finiteness of Walrasian equilibria.

\footnotetext{
${ }^{1}$ By the same token, Allen's result can also be related to deeper economic primitives.

${ }^{2}$ Note that regular maps are trivially of FST.
} 


\section{Notation and preliminary results}

Consider an economy with $L$ commodities $(\ell=1, \ldots, L)$ and $I$ agents $(i=$ $1, \ldots, I)$. Let $\Omega$ be the non-negative orthant of $\mathbb{R}^{L}$ and let each agent $i$ be defined by her endowment $\omega^{i} \in \Omega$ and her preferences $\succsim_{i}$, a complete order on $\Omega$ with the following "rationality" properties:

(P1) completeness and transitivity.

If $x \succsim_{i} y$ and $y \succsim_{i} x$, then $x$ is indifferent to $y$ and we write $x \sim_{i} y$. If $x \succsim_{i} y$ but not $x \sim_{i} y$, then $x$ is strictly preferred to $y$ and we write $x \succ_{i} y$. We call the partial preference order $\succ_{i}$ continuous if it satisfies:

(P2) continuity $\left(\left\{x: y \succ_{i} x\right\}\right.$ and $\left\{y: y \succ_{i} x\right\}$ are open).

In addition, we shall assume strict monotonicity and strict convexity:

(P3) strict monotonicity $\left(x \geq y\left(x_{\ell} \geq y_{\ell}, \forall \ell=1, \ldots, L\right)\right.$ and $x \neq y \Rightarrow x \succ_{i}$ y);

(P4) strict convexity $\left(x \sim_{i} y\right.$ and $\left.x \neq y \Rightarrow \forall \alpha \in(0,1), \alpha x+(1-\alpha) y \succ_{i} x\right)$.

As in Castro et al. (2010), let $\Xi$ denote the space of all such preferences. Furthermore, denote $\mathcal{E} \equiv(\Xi \times \Omega)^{I}$ with typical element $e=\left(\succ^{1}, \omega^{1}, \ldots \succ^{I}\right.$ ,$\left.\omega^{I}\right)$ the space of all $L$ by $I$ economies described by preferences and endowments.

Next, consider the set of smooth (i.e., $C^{\infty}$ ) pure exchange economies with $L$ commodities $(\ell=1, \ldots, L)$ and $I(i=1, \ldots, I)$ agents, generated by each element $e \in \mathcal{E}$ and described by aggregate excess demand (AED) functions $z$ depending smoothly on prices and endowments $\omega \in \Omega \subseteq \mathbb{R}_{+}^{L}$. Normalize prices to lie in the $(L-1)$-dimensional unit simplex $\Delta \equiv\left\{p \in \mathbb{R}_{+}^{L} \mid \sum_{\ell=1}^{L} p_{\ell}=\right.$ $1\}$. Note that an equilibrium price is $p \in \Delta$ such that $z(p, \omega)=0$. We write $z(p, \omega)=z(p)$, when $\omega$ is held fixed.

We further assume the standard Boundary Condition found in Debreu (1970) for at least one consumer (cf. Assumption A therein), in Allen (1984) and in Mas-Colell and Nachbar (1991). We state the Boundary Condition in terms of the AED as in Allen (1984), but the notion is equivalent to that used in Castro et al. (2010):

Definition 2.1. (Boundary Condition) An AED $z$ fulfills the boundary condition (BC), if for every $\omega \in \Omega$ and every sequence $\left(p_{n}\right)_{n \in \mathbb{N}} \in \Delta$ converging to the boundary $\partial \Delta$ as $n \rightarrow \infty,\left\|z\left(p_{n}, \omega\right)\right\|$ is unbounded. 
We denote by $\mathcal{Z}$ the set of smooth AEDs $z: \operatorname{int}(\Delta) \rightarrow \mathbb{R}^{L-1}$, where $\operatorname{int}(\Delta)$ denotes the interior of $\Delta$. Note that if $z$ satisfies (BC) then $z$ is not defined on the boundary of $\Delta$.

The equivalence between AED functions in $\mathcal{Z}$ and the underlying preferences and endowments in $\mathcal{E}$ established in Castro et al. (2010, Corollary 4.7) allows us to work with AEDs directly and still obtain genericity results couched in the more fundamental primitives of preferences and endowment. More formally, the result states:

Theorem 2.2 (Castro et al. (2010)). Let $z_{0}(p) \in \mathcal{Z}$ be the $A E D$ for a $C^{2}$ economy $e_{0} \in \mathcal{E}$ with $L$ goods and $I$ agents characterized by $C^{2}$-preferences $\succ_{0}^{i}$ satisfying $(P 1)-(P 4)$ and $(B C)$ and endowments $\omega_{0}^{i}, i=1, \ldots, I$. An $A E D z(p)$ is a perturbation of $z_{0}(p)$ if and only if $z(p)$ is the $A E D$ for an economy $e \in \mathcal{E}$ with $L$ goods and $I$ agents such that the new preferences $\succ^{1}$ of the first agent are perturbations of $\succ_{0}^{1}$ and the new endowments $\omega^{1}$ are perturbations of $\omega_{0}^{1}$.

\section{Concepts from Differential Topology}

This section recalls some basic concepts from differential topology (see, for example, Golubitsky and Guillemin (1973), Hirsch (1976) or du Plessis and Wall (1995)). Unless explicitly stated to the contrary, manifolds are assumed to have empty boundary.

\subsection{Germs and jets}

In what follows let $f: N \rightarrow P$ be a $C^{\infty}$ map between smooth manifolds of dimension $n$ and $p$, respectively. Denote by $f^{\wedge}$ its germ at $x \in N$, that is, the equivalence class of maps $g: U \rightarrow P$ defined in some neighborhood $U \subset N$ of $x$, that agree with $f$ in a (possibly smaller) neighborhood of $x$. The map $f$ is also often called a representative of the germ $f^{\wedge}$. We often specify source and target values when referring to a germ by writing $f^{\wedge}: N, x \rightarrow P, y$ with $y=f(x)$.

The study of singularities makes ample use of the concept of $k$-jet which we may think of as the $k^{t h}$-order Taylor polynomial of a map $f$ at $x$. We present here the formal definition as in Golubitsky and Guillemin (1973) with adapted notation. 
Definition 3.1 (Golubitsky and Guillemin (1973), Definition 2.1, p. 37). Let $N$ and $P$ be smooth manifolds and $x \in N$. Suppose $f, g: N \rightarrow P$ are smooth maps with $f(x)=g(x)=y$.

1. $f$ has first-order contact with $g$ at $x$ if $(d f)_{x}=(d g)_{x}$ as mappings of $T_{x} N \rightarrow T_{y} P$, the tangent spaces of $N$ and $P$ at $x$ and $y$, respectively.

2. $f$ has $k^{\text {th }}$-order contact with $g$ at $x$ if $(d f): T N \rightarrow T P$ has $(k-1)^{s t}-$ order contact with $(d g)$ at every point in $T_{x} N$. This is written as $f \sim_{k} g$ at $x$.

3. Let $J^{k}(N, P)_{x, y}$ denote the set of equivalence classes under " $\sim_{k}$ at $x$ " of maps $f: N \rightarrow P$ where $f(x)=y$.

4. Let $J^{k}(N, P)=\bigcup_{(x, y) \in N \times P} J^{k}(N, P)_{x, y}$ (disjoint union). An element $j^{k} f \in J^{k}(N, P)$ is called a $k$-jet from $N$ to $P$.

Jet-space $J^{k}(N, P)$ is a smooth vector bundle over $N \times P$, therefore a manifold, and

$$
\operatorname{dim}\left(J^{k}(N, P)\right)=n+p\left(\begin{array}{c}
n+k \\
k
\end{array}\right) .
$$

By taking coordinates, we can identify the fiber $J^{k}(N, P)_{x, y}$ with the space $J^{k}(n, p)$ of jets $\mathbb{R}^{n} \rightarrow \mathbb{R}^{p}$ with 0 source and target. Similarly, the projection on $N$ defines a vector bundle $J^{k}(N, P) \rightarrow N$ and, for a smooth map $f: N \rightarrow$ $P$, we can view its $k$-jet, $j^{k} f$, as a smooth section of this bundle.

\subsection{Whitney and compact-open topologies}

Next we shall describe various standard topologies on $C^{\infty}(N, P)$ (cf. du Plessis and Wall (1995, Section 3.4)). Recall that a topology on a set $X$ can be specified by a subbase, i.e., a collection $\mathcal{B}$ of subsets of $X$, with the property that any open set in $X$ can be written as a union of finite intersections of elements of $\mathcal{B}$.

Definition 3.2. The $C^{0}$ compact-open topology on $C^{k}(P, N)$ is defined by the subbase of sets of the form

$$
A(K, U)=\{f \mid f(K) \subset U\},
$$

where $K \subset N$ is compact and $U \subset P$ is open. 
The $C^{0}$ Whitney topology on $C^{k}(P, N)$ is defined by the subbase of sets of the form

$$
\bigcap_{\alpha} A\left(K_{\alpha}, U_{\alpha}\right)
$$

where $\left\{K_{\alpha}\right\}$ is a locally finite collection of compact subsets $K_{\alpha} \subset N$ and $\left\{U_{\alpha}\right\}$ is a collection of open subsets $U_{\alpha} \subset P$.

The Whitney topology is, in general, finer than the compact-open topology, in the sense that it has more open sets. However, if $N$ is compact, the two topologies are equivalent.

The $C^{0}$ topologies compare values of functions. In order to also compare derivatives up to some finite order, one proceeds as follows: let $k<\infty$ and, as above, view the $k$-jet of a map $f: N \rightarrow P$ as a section of the jet bundle $J^{k}(N, P) \rightarrow N$. Then there is a natural inclusion map

$$
\begin{aligned}
\iota^{k}: C^{\infty}(N, P) & \hookrightarrow C^{\infty}\left(N, J^{k}(N, P)\right), \\
f & \mapsto j^{k} f .
\end{aligned}
$$

Definition 3.3. The $C^{k}$ compact-open topology on $C^{\infty}(N, P)$ is the subspace topology (under the inclusion $\iota^{r}$ ) induced by the $C^{0}$ compact-open topology on $C^{0}\left(N, J^{k}(N, P)\right)$.

The $C^{k}$ Whitney topology on $C^{\infty}(N, P)$ is the subspace topology (under the inclusion $\iota^{r}$ ) induced by the $C^{0}$ Whitney topology on $C^{0}\left(N, J^{k}(N, P)\right)$.

The $C^{\infty}$ compact-open topology and the $C^{\infty}$ Whitney topology on $C^{\infty}(N, P)$ are defined as the unions over all $k$ of the respective $C^{k}$ topologies.

Note that the $C^{k}$ Whitney topology is, in general finer than the $C^{k}$ compact-open topology and that, in both cases, the $C^{l}$ topologies are finer than the $C^{k}$ topologies for $l \geq k$.

Remark 3.4. The jet map

$$
j^{k}: C^{\infty}(N, P) \rightarrow C^{\infty}\left(N, J^{k}(N, P)\right)
$$

is continuous with respect to the $C^{k+l}$-topology on $C^{\infty}(N, P)$ and the $C^{l}$ topology on $C^{\infty}\left(N, J^{k}(N, P)\right)$ for any $l \geq 0$. 


\subsection{Transversality}

In order to prove our main result of this section we need a few transversality results. For completeness, we write them here with adaptations to suit our notation. We use, as is standard, $T_{a} A$ to denote the tangent space, at a point $a$, to a manifold $A$.

Definition 3.5 (Golubitsky and Guillemin (1973, Definition II, 4.1)). Let $N$ and $P$ be manifolds and $f: N \rightarrow P$ be a smooth map. Let $W$ be a submanifold of $P$ and $x$ a point in $N$. We say $f$ intersects $W$ transversally at $x$ (denoted by $f \pitchfork W$ at $x$ ) if either

(a) $f(x) \notin W$, or

(b) $f(x) \in W$ and $T_{f(x)} P=T_{f(x)} W+(d f)_{x}\left(T_{x} N\right)$.

If $A$ is a subset of $N$, we say $f$ intersects $W$ transversally on $A$ if $f \pitchfork W$ for all $x \in A$. We say $f$ intersects $W$ transversally if $f \pitchfork W$ on $N$.

Proposition 3.6 (Golubitsky and Guillemin (1973, Proposition II, 4.2)). Let $N$ and $P$ be smooth manifolds and $W \subseteq P$ a submanifold. Suppose that $\operatorname{dim} N<\operatorname{codim} W$ and let $f: N \rightarrow P$ be smooth. If $f$ intersects $W$ transversally then $f(N) \cap W=\emptyset$.

Theorem 3.7 (Thom Transversality Theorem, Golubitsky and Guillemin (1973, Theorem II, 4.9 and Corollary II, 4.10)). Let $N$ and $P$ be smooth manifolds and $W$ a submanifold of $J^{k}(N, P)$. Let

$$
T_{W}=\left\{f \in C^{\infty}(N, P) \mid j^{k} f \pitchfork W\right\} .
$$

Then $T_{W}$ is a residual subset of $C^{\infty}(N, P)$ in the Whitney $C^{\infty}$ topology. If $W$ is closed then $T_{W}$ is open.

Recall that, in a Baire space, residual implies dense. The space $C^{\infty}(N, P)$ is Baire so, we have that $T_{W}$ is dense in $C^{\infty}(N, P)$.

In order to have genericity results for the $C^{\infty}$ compact-open topology (as well as for the Whitney topology), we next prove a version of Thom Transversality in this setting (cf. Allen (1984, Remark 1)).

Proposition 3.8. Let $N$ and $P$ be smooth manifolds and let $W$ be a closed submanifold of $J^{k}(N, P)$. Let

$$
T_{W}=\left\{f \in C^{\infty}(N, P) \mid j^{k} f \pitchfork W\right\} .
$$


Let $1 \leq l \leq \infty$. Then $T_{W}$ is a residual subset of $C^{\infty}(N, P)$ in the compactopen $C^{k+l}$ topology.

Proof. Cover $N$ by a countable family $\left\{K_{j}\right\}_{j=1}^{\infty}$ of compact sets, such that each $K_{j}$ is the image of a closed ball under some coordinate chart on $N$.

Let $\mathcal{T}_{W, j}$ be the set of maps into jet space which are transverse to $W$ over $K_{j}$, i.e.,

$$
\mathcal{T}_{W, j}=\left\{g \in C^{\infty}\left(N, J^{k}(N, P)\right) \mid g_{\mid K_{j}} \pitchfork W\right\}
$$

and let $T_{W, j}$ be the set of maps in $C^{\infty}(N, P)$ whose $k$-jet is transverse to $W$ over $K_{j}$, i.e.,

$$
\begin{aligned}
T_{W, j} & =\left\{f \in C^{\infty}(N, P) \mid j^{k} f_{\mid K_{j}} \pitchfork W\right\} \\
& =\left(j^{k}\right)^{-1}\left(\mathcal{T}_{W, j}\right) .
\end{aligned}
$$

Then we have

$$
T_{W}=\bigcap_{j=1}^{\infty} T_{W, j} .
$$

It follows from Hirsch (1976, Theorem III, 2.1(b)) (which applies to manifolds with boundary and therefore to $K_{j}$ ) that $\mathcal{T}_{W, j}$ is open in the $C^{l}$ compact-open topology on $C^{\infty}\left(N, J^{k}(N, P)\right)$. Then Remark 3.4 gives that $\mathcal{T}_{W, j}$ is open in $C^{\infty}(N, P)$ in the $C^{k+l}$ compact-open topology. Therefore, we see from (1) that $T_{W}$ is a countable intersection of open subsets in the $C^{k+l}$ compact-open topology.

Finally, by Thom Transversality (Theorem 3.7), $T_{W}$ is dense in the $C^{\infty}$ Whitney topology. Therefore it is dense in the $C^{l}$ compact-open topology for any $l$. This finishes the proof.

\section{Concepts from singularity theory}

This section recalls the concepts from singularity theory necessary for our results. We include statements of the most basic definitions and results for the benefit of the reader less familiar with singularity theory. Our main references are Mather (1968), Gibson et al. (1976), Golubitsky and Guillemin (1973) and du Plessis and Wall (1995). 


\section{1 $\mathcal{K}$-Equivalence}

As before we let $f: N \rightarrow P$ be a $C^{\infty}$ map between smooth manifolds of dimension $n$ and $p$, respectively. Denote by $f^{\wedge}: N, x \rightarrow P, y$ its germ at $x \in N$, with $y=f(x)$. A singularity of $f$ (or of $f^{\wedge}$ ) is a point $x \in N$ for which the Jacobian of $f$ fails to have maximal rank.

The mention of equivalence classes requires a notion of equivalence. Here, we are interested in $\mathcal{K}$ - or contact-equivalence, as in Mather (1968) with adapted notation.

Definition 4.1 (Mather (1968), Definition 2.5, p. 138). Let $C^{\infty}(N, P)_{x, y}$ denote the space of map-germs at $x$. We say that $f^{\wedge}, g^{\wedge} \in C^{\infty}(N, P)_{x, y}$ are contact equivalent if and only if there exists an invertible $C^{\infty}$ map-germ $H^{\wedge}: N \times P,(x, y) \rightarrow N \times P,(x, y)$ such that

(a) $H^{\wedge}$ is surjective

(b) $H^{\wedge}\left(\right.$ graph $\left.f^{\wedge}\right)=\operatorname{graph} g^{\wedge}$.

Let us denote by $\mathcal{K}$ the group of invertible $C^{\infty}$ map-germs $H^{\wedge}: N \times$ $P,(x, y) \rightarrow N \times P,(x, y)$ for which there exists a map-germ $h^{\wedge}: N, x \rightarrow N, x$ such that the following diagram commutes

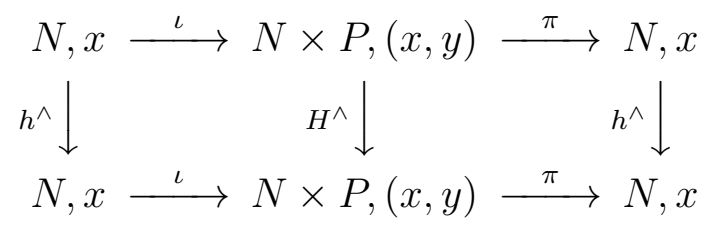

where $\iota$ denotes the germ at $x$ of the inclusion $N \rightarrow N \times P$, and $\pi$ denotes the germ of the projection $N \times P \rightarrow N$. An action of $\mathcal{K}$ on $C^{\infty}(N, P)_{x, y}$ is defined by (1 denotes the identity map-germ $N, x \rightarrow N, x)$

$$
\left(\mathbf{1}, H^{\wedge} \circ f^{\wedge}\right)=H^{\wedge} \circ\left(\mathbf{1}, f^{\wedge}\right) \circ\left(h^{\wedge}\right)^{-1}
$$

and characterized by

$$
H^{\wedge}\left(\operatorname{graph} f^{\wedge}\right)=\operatorname{graph}\left(H^{\wedge} \cdot f^{\wedge}\right),
$$

where $H^{\wedge} \cdot f^{\wedge}$ denotes the action of $\mathcal{K}$ on $C^{\infty}(N, P)_{x, y}$. From here we see that contact equivalence transforms the graph of a germ into the graph of equivalent germs.

We have the following useful result concerning the zeros of $\mathcal{K}$-equivalent map-germs. 
Lemma 4.2. Let $f^{\wedge}: N, x \rightarrow P, y$ be a map-germ. The set of zeros of $f^{\wedge}$ is preserved by $\mathcal{K}$-equivalence.

Proof. Mather (1969, proof of theorem 2.1) shows that there exists a diffeomorphism-germ $h^{\wedge}: N, x \rightarrow N, x$ such that, if $f^{\wedge}, g^{\wedge}: N, x \rightarrow P, y$ are $\mathcal{K}$-equivalent map-germs, then

$$
\left(f^{\wedge} \circ h^{\wedge}\right)^{-1}(0)=\left(g^{\wedge}\right)^{-1}(0) .
$$

Mather (1968) establishes necessary and sufficient conditions for a $C^{\infty}$ map-germ to be finitely determined. A map-germ $f^{\wedge}: N, x \rightarrow P, y$ is finitely determined if there exists an integer $k$ such that any germ $g^{\wedge}: N, x \rightarrow$ $P, y$ with the same $k$-jet as $f^{\wedge}$ is equivalent to $f^{\wedge}$. We say the germ is $k$ determined to specify the order of the Taylor polynomial and, if there is a need for extra clarity, we may say $k-\mathcal{K}$-determined. Finally, a germ is said to be finitely $\mathcal{K}$-determined if it is $k-\mathcal{K}$-determined for some $k$.

\subsection{Finite Singularity Type}

For the concept of finite singularity type (FST) we refer to du Plessis and Wall (1995, Section 2.4) where several equivalent versions are given. We shall adopt the following:

Definition 4.3. A map-germ $f^{\wedge}: N, x \rightarrow P, y$ is of finite singularity type if and only if it is finitely $\mathcal{K}$-determined.

The characterization of FST is done best by resorting to auxiliary subsets of jet-space. We shall not give the definition of these sets as it would require further non-trivial concepts from singularity theory but, instead, use their properties which are given, at the local level, in du Plessis and Wall (1995, p. 30). We know then that, at the local level, there exist algebraic sets $W^{k}(n, p) \subseteq J^{k}(n, p)$ satisfying the following properties:

1. $W^{k}(n, p)$ is closed;

2. if there exists $k$ such that $j^{k} f(x) \notin W^{k}(n, p)$ then $f^{\wedge}$ is $k$ - $\mathcal{K}$-determined;

3. $\operatorname{codim}_{J^{k}(n, p)} W^{k}(n, p) \longrightarrow \infty$ as $k \rightarrow \infty .^{3}$

\footnotetext{
${ }^{3}$ This property goes back to Tougeron (1972, Lemma VII, 5.3).
} 
From property 2 it follows that if there exists $k$ such that $j^{k} f^{\wedge}(x) \notin$ $W^{k}(n, p)$ then $f^{\wedge}$, the germ of $f$ at $x$, is of FST.

At global level, we consider $C^{\infty}(N, P)$. We have a jet-bundle $J^{k}(N, P) \rightarrow$ $N \times P$ with fiber over $(x, y) \in N \times P$

$$
J^{k}(N, P)_{(x, y)} \simeq J^{k}(n, p) .
$$

We have a subbundle

$$
W^{k}(N, P) \subseteq J^{k}(N, P)
$$

defined by $W^{k}(n, p) \subseteq J^{k}(n, p)$. By property $1, W^{k}(N, P) \subseteq J^{k}(N, P)$ is closed and by property 3 , since $\operatorname{codim}_{J^{k}(N, P)} W^{k}(N, P)=\operatorname{codim}_{J^{k}(n, p)} W^{k}(n, p)$,

$$
\lim _{k \rightarrow \infty} \operatorname{codim}_{J^{k}(N, P)} W^{k}(N, P)=\infty .
$$

From the definition of the $W^{k}(N, P)$ (du Plessis and Wall (1995, p. 30)) one has that $W^{k}(N, P) \supseteq W^{k+1}(N, P) \supseteq \cdots$. We can therefore state the following:

Lemma 4.4. If there exists $k$ such that $j^{k} f(x) \notin W^{k}(N, P)$ for all $x \in N$ then $f^{\wedge}$ is of FST for all $x \in N$.

Proof. The result follows from local property 2 .

We note that the hypothesis in Lemma 4.4 is weaker than demanding global FST (see du Plessis and Wall (1995) for the definition) but stronger than asking for $f^{\wedge}$ to be of FST at every point of $N$.

Remark 4.5. Even for polynomial maps, the concept of FST is fairly subtle, and to check whether a given map germ is of FST, one resorts to the methods of Bruce et al. (1987). Thus, for example, the germ at $(0,0)$ of the map

$$
f(x, y)=\left(x y, x^{2}\right)
$$

is not of FST (as also follows from Theorem 5.5 below), while the germ at $(0,0)$ of the map

$$
g(x, y)=\left(x y, x^{2}+y^{4}\right)
$$

is of FST (and indeed has $(0,0)$ as an isolated zero). 


\section{$5 \quad$ Results}

\subsection{Genericity of FST-maps}

We can now state and prove the first main result, establishing that FST is a generic (valid in an open and dense set) property of maps (cf. du Plessis and Wall (1995, Section 2.4)).

Theorem 5.1. The set

$$
C_{F S T}^{\infty}=\left\{f \in C^{\infty}(N, P) \mid f^{\wedge} \text { is of FST for all } x\right\}
$$

contains an open and dense subset in the Whitney $C^{\infty}$ topology which is residual (therefore, dense) in the compact-open $C^{\infty}$ topology.

Proof. Let $k_{0}$ be such that $\operatorname{dim} N<\operatorname{codim} W^{k}(N, P)$ for $k \geq k_{0}$. Then, by Proposition 3.6, we have that

$$
j^{k} f(N) \cap W^{k}(N, P)=\emptyset,
$$

if and only if

$$
j^{k} f \pitchfork W^{k}(N, P) \text {. }
$$

Let $S_{k}=\left\{f \in C^{\infty}(N, P) \mid j^{k} f \pitchfork W^{k}(N, P)\right\}$. Thom Transversality (Theorem 3.7) implies that $S_{k} \subseteq C^{\infty}(N, P)$ is open and dense in the Whitney $C^{\infty}$ topology provided $k \geq k_{0}$. By Lemma 4.4, if $f \in S_{k}$, then $f^{\wedge}$ is of FST for all $x \in N$, so $f \in C_{F S T}^{\infty}$. We have then proved that

$$
S_{k} \subseteq C_{F S T}^{\infty}
$$

Because when a map is $k$-determined, it is also $(k+1)$-determined, we have

$$
C_{F S T}^{\infty} \supseteq \bigcup_{k \geq k_{0}} S_{k}
$$

The union of open and dense sets is itself an open and dense set.

Finally, to obtain the result for the compact-open topology, we apply the same argument, but now using Proposition 3.8 instead of the Thom Transversality Theorem. 
Remark 5.2. In the present case (when the dimensions of $N$ and $P$ are the same) this approach is not applicable to showing genericity of just regular maps. Indeed, $f \in C^{\infty}(N, P)$ is regular if and only if $j^{1} f(N)$ avoids the subspace $W$ of $J^{1}(N, P)$ given by the vanishing of the determinant of the Jacobian matrix. But the codimension of $W$ is one, which is never greater than the dimension of $N$. Therefore, Proposition 3.6 cannot be applied.

One may (and, in fact, we shall do so in Section 5.3) ask whether it is possible to perturb a given map to being of FST without changing its 1-jet. This is a relevant point for applications and the answer is affirmative as we show next.

Theorem 5.3. Let $f_{0}: N \rightarrow P$. There exists $f: N \rightarrow P$ of FST arbitrarily (both in the Whitney and compact-open $C^{\infty}$ topologies) close to $f_{0}$ and such that $j^{1} f=j^{1} f_{0}$.

Proof. Consider the projection

$$
\pi: J^{k}(N, P) \rightarrow\left(J^{k} / J^{1}\right)(N, P)
$$

where $\left(J^{k} / J^{1}\right)(N, P)$ is the quotient bundle corresponding to jets of degree $k$ whose linear and constant parts vanish. Denote by $\tilde{j}^{k} g$ the projection of $j^{k} g$ in $\left(J^{k} / J^{1}\right)(N, P)$.

Let $W^{k}(N, P) \subset J^{k}(N, P)$ be as defined in (2) and let

$$
\tilde{W}^{k}(N, P)=\pi\left(W^{k}(N, P)\right) \subset\left(J^{k} / J^{1}\right)(N, P) .
$$

Then

$$
\lim _{k \rightarrow \infty} \operatorname{codim}_{\left(J^{k} / J^{1}\right)(N, P)} \tilde{W}^{k}(N, P)=\infty .
$$

Fix $f_{0}$. It suffices to prove the result for the Whitney topology, since it is finer than the compact-open topology. Proceed as in the proof of density of Theorem II, 4.9 in [10] but take the space of perturbations $B^{\prime}$ to be the space of polynomial maps $\mathbb{R}^{n} \rightarrow \mathbb{R}^{p}$ of degree $k$ with vanishing linear and constant terms. Note that $g_{0}=f_{0}$. Furthermore, this new $B^{\prime}$ corresponds exactly to the fibers of $\left(J^{k} / J^{1}\right)(N, P)$.

The remaining part of the proof goes through to show that there exists a neighborhood $B \subset B^{\prime}$ of $0 \in B^{\prime}$ such that

$$
\left\{b \in B \mid \tilde{j}^{k} g_{b} \pitchfork \tilde{W}^{k}(N, P)\right\}
$$


is dense in $B$. Because of (3), for $k$ sufficiently large, $\tilde{j}^{k} g_{b} \pitchfork \tilde{W}^{k}(N, P)$ means that

$$
\tilde{j}^{k} g_{b} \cap \tilde{W}^{k}(N, P)=\emptyset .
$$

In other words, $g_{b}$ is of FST (it is in fact $k$-determined, cf. local property 2 in Section 4.2).

Remark 5.4. The previous theorem holds if we change $j^{1} f=j^{1} f_{0}$ to $j^{r} f=$ $j^{r} f_{0}$ for any fixed $r$. The proof is analogous.

\subsection{Local Isolation of Zeros}

In order to relate FST to the set of zeros of a map, we need to consider both critical and regular zeros. This is because, from the point of view of applications to the study of AEDs, we are interested in the case when $N$ and $P$ are equidimensional $(n=p)$.

In fact, if $n<p$ then all zeros are critical and we can refer to du Plessis and Wall (1995, p. 30) to conclude that the set of zeros is finite, provided $f$ is of FST: they show that $\Sigma(f) \cap f^{-1}(y)(y \in P)$ is finite, where $\Sigma(f)$ is the set of all critical points. The case $n>p$ is of no interest since no zeros, critical or regular, are isolated.

In the equidimensional case, we can still refer to du Plessis and Wall (1995, p. 30) to get finiteness of the critical zeros. However, even though regular zeros are isolated, this does not rule out the existence of a sequence of regular zeros converging to a critical one (and this is the reason why MasColell and Nachbar (1991, Corollary 3) only obtain countability, rather than finiteness, of equilibria). As we shall show, in the present context of FST maps, this situation cannot occur.

In view of Lemma 4.2, it is enough to state and prove the result for polynomial maps since $f^{\wedge}$ being of FST implies that $f^{\wedge}$ is $\mathcal{K}$-equivalent to its $k$-jet, a polynomial of degree $k$. This reduction is crucial, since it allows for the application of the Curve Selection Lemma in the proof below.

Theorem 5.5. Let $M$ and $P$ be manifolds of the same dimension and let $f$ : $M \rightarrow P$ be a polynomial map. Assume $f^{\wedge}$ is of FST for all $x \in Z=f^{-1}(0)$. Then the critical zeros of $f$ are isolated in $M$.

Proof. We proceed by contradiction. Let $p$ be a critical zero, that is, $p \in$ $\Sigma(f)$. Assume that for all neighborhoods $V$ of $p$ in $M$ there exists a regular 
zero $x$ of $f$ in $V$. The Curve Selection Lemma (see Milnor, 1968, Lemma 3.1) implies that there exists an analytic curve $\gamma:[0, \epsilon[\rightarrow M$ with $\gamma(0)=p$ and such that $\gamma(t)$ is a regular zero for $t>0$. But, since $\gamma$ is a curve of zeros of $f$, it is contained in $\Sigma(f)$. This contradicts the construction of $\gamma$. We can therefore assert that there exists a neighborhood $V$ of $p$ in $M$ such that

$$
V \cap \Sigma(f) \cap f^{-1}(0)=V \cap f^{-1}(0) .
$$

Because $f$ is of FST, $\Sigma(f)$ must be finite and $V \cap f^{-1}(0)$ contains only finitely many zeros. Hence, points in $\Sigma(f) \cap f^{-1}(0)$ are locally isolated.

Corollary 5.6. Let $N$ and $P$ be smooth manifolds of the same dimension and let $f: N \rightarrow P$ be a smooth map such that the germ $f^{\wedge}$ is of FST for all $x \in N$. Then the zeros of $f$ are locally isolated. If, moreover, $f$ is proper then the set of its zeros is finite.

Proof. By Lemma 4.2 we may assume that $f^{\wedge}$ is polynomial and of FST in a neighborhood $M$ of each point in $N$. Applying the result of du Plessis and Wall (1995, p. 30) cited above and Theorem 5.5 in such a neighborhood around each critical zero, we see that all critical zeros of $f$ are isolated in $N$. We also know that the regular zeros are locally isolated $\operatorname{since} \operatorname{dim} N=\operatorname{dim} P$. Finally, when $f$ is proper, $f^{-1}(0)$ is compact, and local isolation implies finiteness.

\subsection{Finiteness of Walrasian equilibria}

In this section we use the genericity of FST maps to prove our main results on finiteness of equilibria. The concept of FST is essential here since, in the present setup of AEDs, we are in the equidimensional case where there is no corresponding genericity result for regular economies (see Remark 5.2).

In order to apply the results in the previous section to AEDs, we want to think of $N=\operatorname{int}(\Delta)$ and $P=\mathbb{R}^{L-1}$. These are smooth manifolds but $\operatorname{int}(\Delta)$ is a non-compact manifold and we therefore need the next result before being able to prove finiteness of Walrasian equilibria.

Proposition 5.7. Let $z: \operatorname{int}(\Delta) \rightarrow \mathbb{R}^{L-1}$ be an AED satisfying the Boundary Condition ( $B C)$. Then $z$ is proper.

Proof. We need to show that for any convergent sequence in the target, any sequence of pre-images in the source has a convergent subsequence. 
For a convergent sequence in $\mathbb{R}^{L-1}$, we take the corresponding sequence $\left(p_{n}\right)_{n \in \mathbb{N}}$ in $\operatorname{int}(\Delta)$. Since $\Delta$ is compact $\left(p_{n}\right)_{n \in \mathbb{N}}$ has a convergent subsequence, say $\left(p_{n_{i}}\right)_{n_{i} \in \mathbb{N}}$. This subsequence converges to a point in $\operatorname{int}(\Delta)$. Otherwise, $\left(p_{n_{i}}\right)_{n_{i} \in \mathbb{N}}$ would converge to the boundary of $\Delta$ and by $(\mathrm{BC})$ the original sequence of images in $\mathbb{R}^{L-1}$ would be unbounded.

The following Proposition is now an immediate consequence of Corollary 5.6 .

Proposition 5.8. Let $z: \operatorname{int}(\Delta) \rightarrow \mathbb{R}^{L-1}$ be an AED, of FST, and satisfying the Boundary Condition (BC). Then $z$ has finitely many zeros.

We have seen in Theorem 5.1 that the set of maps of FST is generic in the set of all smooth maps, meaning that it is residual for the $C^{\infty}$ compact-open topology and contains an open and dense set in the $C^{\infty}$ Whitney topology. Applying this to the space of maps $\mathcal{Z}$ representing AEDs we obtain that the subset of $\mathcal{Z}$ consisting of AEDs of FST is generic. Therefore, Proposition 5.8 implies the following main result, where generic is to be understood in the above sense.

Theorem 5.9. The number of equilibria is finite in the economies defined by generic $A E D$ s in $\mathcal{Z}$ satisfying the Boundary Condition (BC).

Applying the result of Allen (1984) in the present setting of unparametrized economies gives finiteness for a residual subset of AEDs in the $C^{\infty}$ Whitney topology (rather than for an open-dense one). Thus, in this setting, our genericity result is stronger.

One the other hand, our result is stronger than that of Mas-Colell and Nachbar (1991, Corollary 3) in that we obtain finiteness, rather than countability, of equilibria for the generic economy.

We further strengthen our result by concluding the paper with the following fact: FST (and thus finiteness of equilibria) is not only a generic property among smooth exchange economies, it is even a generic property among critical smooth exchange economies. In other words, any critical economy can be perturbed to become of FST, while remaining critical.

Theorem 5.10. The subset of FST critical economies is open and dense inside the set of all critical economies in the Whitney $C^{\infty}$ topology and residual in the compact-open $C^{\infty}$ topology. Hence the number of equilibria of the generic critical economy is finite. 
Proof. It follows as a corollary of Theorem 5.3. Note that only density needs proof, as openness (respectively residuality) follows from the genericity Theorem 5.1.

Acknowledgements: Much progress on this paper was made while the first and third authors were visiting QGM at the Department for Mathematical Sciences of the University of Aarhus, Denmark, whose hospitality is gratefully acknowledged. The results found their present shape through various conversations with and suggestions from Andrew du Plessis, to whom the authors express their gratitude.

The authors also wish to thank Beth Allen, Marcus Berliant, Jean-Marc Bonnisseau, Marcel Bökstedt, Gaël Giraud, and John Nachbar for helpful conversations, and in particular, Marty Golubitsky for introducing the first two authors to each other.

\section{References}

[1] Allen, B. (1984): On the finiteness of the equilibrium price set, CARESS Working Paper \#85-03, University of Pennsylvania.

[2] Allen, B. (1994): Randomization and the limit points of monopolistic competition, Journal of Mathematical Economics 23, 205-218.

[3] Berliant, M. and S. Dakhlia (2002): Sensitivity analysis for computable general equilibrium models in the presence of multiple Walrasian equilibria, Economic Theory 19, 459-476.

[4] Bruce, J.W., A.A. du Plessis and C.T.C. Wall (1987): Determinacy and unipotency, Invent. Math. 88 (1987), no. 3, 521-554.

[5] Castro, S.B.S.D., S. Dakhlia, and P. Gothen (2010): Direct perturbations of aggregate excess demand functions, Journal of Mathematical Economics, forthcoming.

[6] Castro, S.B.S.D. and S. Dakhlia, (2008): Thom-Boardman stratification of aggregate excess demand and finiteness of equilibria, SSRN Working Paper 1156100. 
[7] Debreu, G. (1970): Economies with a finite set of equilibria, Econometrica $38,387-392$.

[8] du Plessis, A.A. and C.T.C. Wall (1995): The Geometry of Topological Stability, OUP.

[9] Gibson, C.G., K. Wirthmüller, A.A. du Plessis and E.J.N. Looijenga (1976): Topological Stability of Smooth Mappings, LNM 552.

[10] Golubitsky, M. and V. Guillemin (1973): Stable Mappings and their Singularities, Springer Verlag, New York.

[11] Hirsch, M.W. (1976): Differential topology, Graduate Texts in Mathematics, No. 33, Springer-Verlag, New York-Heidelberg.

[12] Mas-Colell, A (1985), The Theory of General Economic Equilibrium: a Differentiable Approach, Cambridge University Press.

[13] Mas-Colell, A. and J.H. Nachbar (1991): On the finiteness of the number of critical equilibria, with an application to random selections, Journal of Mathematical Economics 20 (1991), 397-409.

[14] Mather, J. (1968): Stability of $C^{\infty}$ maps, III. Finitely determined mapgerms, Publ. Math. IHES, 35,127-156.

[15] Mather, J. (1969): Stability of $C^{\infty}$ maps, IV. Classification of stable germs by $R$-algebras, Publ. Math. IHES, 37,223-248.

[16] Mather, J. (1973): On Boardman Singularities, in Dynamical Systems, M. Peixoto (ed.), 233-248, Academic Press, New York.

[17] Milnor, J. (1968): Singular points of complex hypersurfaces, Princeton University Press.

[18] Tougeron, J.C. (1972): Idéaux de fonctions différentiables, Springer. 